\title{
Research Update
}

\section{When Parents Ask . . . about Preterm Birth, Breastfeeding Success, Breast Cancer, or Waterbirth}

\author{
Mary Lou Moore, PhD, RNC, LCCE, FACCE, FAAN
}

MARY LOU MOORE is an associate professor in the Department of Obstetrics and Gynecology at the Wake Forest University School of Medicine in Winston-Salem, North Carolina.

\begin{abstract}
In this column, the author reviews research on four separate topics: the prevention of preterm birth, the effect of epidurals on breastfeeding success, the possible protective effect of lactation on breast cancer, and laboring in water. In two separate studies-a multisite study in the United States and a second study from Brazil-natural progesterone was shown to significantly reduce the incidence of preterm birth in women at high risk. A third study conducted in the United Kingdom examined the effect of clindamycin on preventing infection that can lead to preterm birth. A group of studies related to lactation found that early breastfeeding was more successful in women who did not have epidural anesthesia. In a Korean study, the lifetime duration of breastfeeding was associated with a significant reduction in breast cancer. Concerning waterbirth, Swiss researchers found that, when a woman labored in water, she regulated both water temperature and bathing duration to ensure that her body temperature and that of the fetus remained within a normal physiological range.

Journal of Perinatal Education, 12(4), 40-43; preterm birth, epidurals, breastfeeding success, breast cancer, waterbirth.
\end{abstract}

I usually think of the research that is reviewed in this column in relation to questions that participants ask in Lamaze classes. In this column, I have posed questions that are relevant to the research described.

\section{Prevention of Preterm Birth}

\section{Question}

My last baby was born at 32 weeks. Is there any way to prevent that from happening again? 


\section{Research Review}

Preterm birth remains a major reason for poor pregnancy outcome. Unfortunately, rates of preterm birth are rising in the United States. Two major reasons for this increase may be that women are choosing pregnancy at later ages and accepting assisted reproductive technology.

Two recent studies-one multisite study by the Maternal-Fetal Medicine Units Network of the National Institute of Child Health and Human Development and another study conducted in Brazil-suggest that one possible solution for at least some preterm labor and birth incidents is the administration of natural progesterone (17 alpha-hydroxyprogesterone caproate, called $17 P)$. Both studies, although using somewhat different approaches, showed a statistically significant decrease in preterm births.

In the United States study headed by Dr. Paul Meis, 463 women with singleton gestations in the current pregnancy and at least one previous preterm birth were initially enrolled (Meis et al., 2003). The oversight committee halted the study because the results were so dramatically different in the intervention group, which received $17 \mathrm{P}$, that committee members believed women in the control group were put at unnecessary risk, leaving a study sample of 351 participants. Births less than 37 weeks gestation were $36.3 \%$ in the intervention group and $54.9 \%$ in the placebo group $(p=.0001)$. Births prior to 32 weeks gestation were $11.4 \%$ in the intervention group and $19.6 \%$ in the placebo group $(p=.0180)$. The progesterone was administered as weekly injections, beginning at 16-20 weeks gestation and continuing until 36 weeks gestation.

In the study conducted in Brazil, women with a wider group of risk factors were included and the progesterone was administered by a daily vaginal suppository ( $\mathrm{Da}$ Fonseca, Bittar, Carvalho, \& Zugaib, 2003). Preterm birth rates less than 37 weeks gestation were $13.8 \%$ for the intervention group and $28.5 \%$ for the placebo group;

Births less than 37 weeks gestation were $36.3 \%$ in the intervention group, which received $17 P$, and $54.9 \%$ in the placebo group. at $<34$ weeks gestation, the rates were $2.7 \%$ for the intervention group and $18.5 \%$ for the placebo group. While further studies with other groups of women are indicated, the results of the Brazilian study offer an encouraging addition to strategies addressing the complex puzzle of premature births.

Infections, particularly genitourinary-tract infections, have long been associated with preterm birth. A second pharmacological agent, the antibiotic clindamycin, was examined in a study from the United Kingdom (Ugwumadu, Manyonda, Reid, \& Hay, 2003). The researchers screened 6,120 pregnant women on their first prenatal visit (between 12 and 22 weeks gestation) for bacterial vaginosis or abnormal vaginal flora. The 494 women with one of these conditions were randomly assigned to receive either oral clindamycin or placebo for five days. Women receiving clindamycin had $10.4 \%$ fewer miscarriages and preterm births (births between 24 and 37 weeks gestation; $p=.0003$ ) than women receiving placebo.

It is important to recognize that, in all of the studies reviewed above, intervention was not successful in all of the women. Preterm birth is a multifactoral, complex issue; any single intervention unlikely will be successful in all women. Nevertheless, data from these studies suggest interventions that may make a difference for some women and their infants, which is encouraging to note.

\section{Epidurals and Breastfeeding Success}

\section{Question}

If I have an epidural, will that affect breastfeeding?

\section{Research Review}

In a study specifically designed to examine the effects of epidural anesthesia during labor on breastfeeding success and bottle supplementation, 115 mother-infant pairs in which the mother received epidural anesthesia were compared with 116 pairs in which the mother received no epidural anesthesia (Baumgarder, Muehl, Fischer, \& Pribbenow, 2003). All of the infants were healthy, fullterm, and born vaginally. The primary outcome of interest included two successful breastfeeding encounters by 24 hours of age, defined as having a LATCH score of 7 or greater. (LATCH scoring awards two points for each of five indicators-latch, audible swallow, type of nipple,

The Journal of Perinatal Education Vol. 12, No. 4, 2003 
comfort, and hold-much as an APGAR score awards two points for each of five indicators of infant response at birth). In the hospital where this study was conducted, nurses routinely include a LATCH score in their clinical assessment and record it for all mother-infant pairs.

The study found that breastfeeding was relatively successful in both groups, but more successful in women who did not have epidurals: $69.6 \%$ of the epidural group and $81 \%$ of the no-epidural group had two successful breastfeeding experiences (Baumgarder et al., 2003). Unfortunately, percentages were not given for the likelihood of receiving bottle supplementation during hospitalization, but the difference between the groups was stated as $<.001$. The differences remained when mother-infant pairs were stratified by parity, use of narcotics, early initiation of breastfeeding, and maternal age.

The study's findings indicated that $69.6 \%$ of the epidural group and $81 \%$ of the no-epidural group had successful breastfeeding experiences.

The reason for these differences was not clear from the data. Baumgarder and colleagues (2003) speculated the reasons might have been multifactoral, including mild neural-behavioral effects of epidural anesthesia and/or real or perceived labor difficulties. The design of the study precluded answering some questions that clearly need to be addressed. For example, did the women who chose to have epidural anesthesia have longer and/or more difficult labors, or did they perceive their labors to be more difficult? Clearly, additional studies will be necessary to further our understanding of the relationship between epidural anesthesia and breastfeeding.

\section{Lactation's Effect on Breast Cancer}

\section{Question}

I've heard that breastfeeding reduces your risk of breast cancer. Is that true?

\section{Research Review}

The possible protective effect of lactation on breast cancer has been a focus of some researchers for more than
20 years. While no studies have found a detrimental effect, research outcomes have varied in the finding of an inverse relationship (i.e., a lower incidence of breast cancer in women who breastfeed).

A recent study from Korea was designed to clarify the inconsistencies in previous studies (Lee, Kim, M., Kim, S., Song, \& Yoon, 2003). The sample for the study was derived from the largest Korean health insurance group that provides services to government employees, teachers, and their family members. The sample included 110,604 premenopausal women, aged 20 years or older, who worked as government employees in 1994 and had been pregnant at least once. In this cohort, $51.9 \%$ reported breastfeeding: 79\% breastfed from 1-12 months, $13 \%$ breastfed from $13-24$ months, and $8 \%$ breastfed for more than 24 months. During the five-year followup period (1995-2000), 360 cases of breast cancer occurred, with 24 deaths. When compared to women who had never breastfed, the relative risk of breast cancer was 0.8 for women who had breastfed for 1-12 months $(p=.096), 0.7$ for women who had breastfed for 13-24 months ( $p=.037)$, and 0.5 for women who had breastfed for more than 24 months ( $p=.003$ ). Thus, no statistically significant difference was evident for those who breastfed for a total of 12 months or less (meaning, the difference could be due to chance). However, a statistically significant difference was evident for those women who breastfed for more than 12 months, with the greatest decrease in risk for those who breastfed for the longest period. The duration of breastfeeding refers to lifetime breastfeeding, not to breastfeeding duration of a single infant. The follow-up period for this study was only five years; therefore, it is certainly possible, and even likely, that additional women in the study will have breast cancer at some time in their lives.

\section{Laboring in Water}

\section{Question}

What is the ideal temperature for laboring in water? How long can I stay in the water?

\section{Research Review}

A group of researchers in Switzerland conducted a study on waterbirth, examining the need for guidelines for water temperature and bathing time (Geissbuehler, Eber- 
The woman regulated both water temperature and bathing duration to ensure that the body temperatures of both mother and child remained within the physiological range.

hard, \& Lebrecht, 2002). Over an eight-year period, 10,775 women with both land births and waterbirths were followed. Body temperature did not differ between the two groups, except at the time of birth when maternal temperature of those mothers who labored in water was $36.9^{\circ} \mathrm{C}\left(98.42^{\circ} \mathrm{F}\right)$ compared to $36.34^{\circ} \mathrm{C}\left(97.3^{\circ} \mathrm{F}\right)$ for mothers who labored on land. Neonatal rectal temperatures did not differ significantly between the two groups.

Geissbuehler and colleagues (2002) concluded that waterbirths posed no thermal risk. The woman regulated both water temperature and bathing duration to ensure that the body temperatures of both mother and child remained within the physiological range. Therefore, the researchers stated, "Cumbersome guidelines for water temperature and bathing time are not only superfluous, but could even dangerously disrupt the physiologic control of temperature regulation" (Geissbuehler et al., 2002, p. 376).

A previous issue of this journal features another "Research Update" column that reviews studies on the safety and efficacy of waterbirth from the Frauenfeld Clinic for Obstetrics and Gynecology in Switzerland (Moore, 2001). Evidence was presented showing slightly lower cesarean birth rates for waterbirths, as well as lower epidural rates and a large drop in episiotomy rates. Subjects were 344,328 women in Swiss clinics from 1986-1997 (Geissbuhler \& Eberhard, 2000).

\section{References}

Baumgarder, D., Muehl, P., Fischer, M., \& Pribbenow, B. (2003). Effect of labor epidural anesthesia on breastfeeding of healthy full-term newborns delivered vaginally. Journal of the American Board of Family Practice, 16, 7-13.

Da Fonseca, E., Bittar, R., Carvalho, M., \& Zugaib, M. (2003). Prophylactic administration of progesterone by vaginal suppository to reduce the incidence of spontaneous preterm birth in women at increased risk: A randomized placebocontrolled double blind study. American Journal of Obstetrics and Gynecology, 188, 419-424.
Geissbuhler, V., \& Eberhard, J. (2000). Waterbirths: A comparative study. Fetal Diagnosis and Therapy, 15(5), 291-300.

Geissbuehler, V., Eberhard, J., \& Lebrecht, A. (2002). Waterbirth: Water temperature and birthing time-Mother knows best. Journal of Perinatal Medicine, 30, 371-378.

Lee, S., Kim, M., Kim, S., Song, M., \& Yoon, S. (2003). Effect of lifetime lactation on breast cancer risk: A Korean women's cohort study. International Journal of Cancer, 105, 390-393.

Meis, P., Klebanoff, M., Thom, E., Dombrowski, M. P., Sibai, B., Moawad, A. H., et al. for the National Institute of Child Health and Human Development Maternal-Fetal Medicine Units Network. (2003). Prevention of recurrent preterm delivery by 17 alpha-hydroxyprogesterone caproate. New England Journal of Medicine, 348, 2379-2385.

Moore, M. L. (2001). Research update: Adopting birth philosophies to guide successful birth practices and outcomes. Journal of Perinatal Education, 10(2), 43-45.

Ugwumadu, A., Manyonda, I., Reid, F., \& Hay, P. (2003). Effect of early oral clindamycin on late miscarriage and preterm delivery in asymptomatic women with abnormal vaginal flora and bacterial vaginosis: A randomized controlled trial. Lancet, 361, 983-988. 